\title{
Nanocarrier drugs in the treatment of brain tumors
}

\author{
Tereza Cerna ${ }^{1,4}$, Marie Stiborova ${ }^{1}$, Vojtech Adam², Rene Kizek ${ }^{3}$, Tomas Eckschlager ${ }^{4}$ \\ ${ }^{1}$ Department of Biochemistry, Faculty of Science, Charles University, Albertov 2030, CZ-128 40 Prague 2, Czech Republic. \\ ${ }^{2}$ Department of Chemistry and Biochemistry, Laboratory metallomics and nanotechnology, Mendel University in Brno and Central European Institute of \\ Technology, Brno University of Technology, Zemédělská 1, CZ-613 00 Brno, Czech Republic. \\ ${ }^{3}$ Department of Human Pharmacology and Toxicology, Faculty of Pharmacology, University of Veterinary and Pharmaceutical Sciences Brno, Palackeho 1, \\ CZ 61242 Brno, Czech Republic. \\ ${ }^{4}$ Department of Pediatric Hematology and Oncology, 2nd Faculty of Medicine, Charles University, and University Hospital Motol, V Uvalu 84 , CZ-150 06 \\ Prague 5, Czech Republic.
}

Correspondence to: Prof. Tomas Eckschlager, Department of Pediatric Hematology and Oncology, 2nd Faculty of Medicine, Charles University, and University Hospital Motol, V Uvalu 84, CZ-150 06 Prague 5, Czech Republic. E-mail: tomas.eckschlager@lfmotol.cuni.cz

How to cite this article: Cerna T, Stiborova M, Adam V, Kizek R, Eckschlager T. Nanocarrier drugs in the treatment of brain tumors. J Cancer Metastasis Treat 2016;2:407-16.

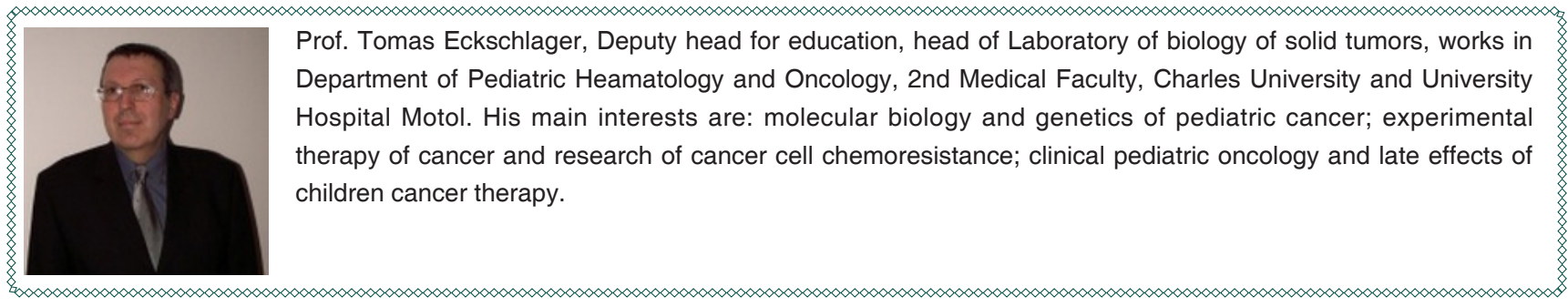

Article history:

Received: $23-12-2015$

Accepted: 25-09-2016

Published: 31-10-2016

\section{Key words:}

Brain tumors,

nanoparticles,

enhanced permeability and

retention effect,

active targeting,

blood-brain barrier

\begin{abstract}
Nanoparticle-mediated targeted delivery of drugs might significantly reduce the dosage and optimize their release properties, increase specificity and bioavailability, improve shelf life, and reduce toxicity. Some nanodrugs are able to overcome the blood-brain barrier that is an obstacle to treatment of brain tumors. Vessels in tumors have abnormal architecture and are highly permeable; moreover, tumors also have poor lymphatic drainage, allowing for accumulation of macromolecules greater than approximately $40 \mathrm{kDa}$ within the tumor microenvironment. Nanoparticles exploit this feature, known as the enhanced permeability and retention effect, to target solid tumors. Active targeting, i.e. surface modification of nanoparticles, is a way to decrease uptake in normal tissue and increase accumulation in a tumor, and it usually involves targeting surface membrane proteins that are upregulated in cancer cells. The targeting molecules are typically antibodies or their fragments; aptamers; oligopeptides or small molecules. There are currently several FDA-approved nanomedicines, but none approved for brain tumor therapy. This review, based both on the study of literature and on the authors own experimental work describes a comprehensive overview of preclinical and clinical research of nanodrugs in therapy of brain tumors.
\end{abstract}




\section{INTRODUCTION}

Brain tumors are divided into two groups: (i) primary, originating and residing within the brain and (ii) secondary (metastatic), originating from a primary cancer outside the central nervous system and spreading into the brain. Metastatic tumors are more frequent than primary tumors in adult patients while primary ones are the most frequent solid tumors of childhood. The histological spectrum of brain tumors in children and adolescents differs from that in adults. ${ }^{[1]}$

Primary brain tumors represent a heterogeneous group as classified according to WHO. According to the Central Brain Tumor Registry of the United States (CBTRUS) 2005-2009 report, the incidence in the US of CNS tumors was 20.6 cases per 100,000 persons/year, the incidence of malignant tumors was $7.3 / 100,000$ persons/year and the incidence of low-grade tumors was $13.3 / 100,000$ persons/year. ${ }^{[2]}$

The most frequent brain tumors in all age groups are tumors originating from glial cells - gliomas that represent a wide spectrum of tumors ranging from slow growing to highly aggressive tumors. WHO classifies gliomas within four grades: grade I (pilocytic astrocytoma), grade II (diffuse astrocytoma), grade III (anaplastic astrocytoma), and grade IV (glioblastoma multiforme). The grade III and IV are considered highgrade gliomas (malignant gliomas) and are associated with very poor prognosis. In particular, 5 year survival rate of glioblastoma multiforme, which accounts for half of primary brain tumors, is less than $10 \% .^{[3]}$ Brain metastases are the most common intracranial tumors in adults, with more than 150,000 cases in the USA. In adults with cancer, 8-10\% develop brain metastases, although the incidence of metastases varies considerably among different primary tumor types. Lung, breast, colorectal, renal cell cancer or melanoma can metastasize to the brain and $70 \%$ of brain metastases are due to lung and breast cancer. ${ }^{[4,5]}$ High-grade brain tumors, such as glioblastoma, and brain metastases are often lethal because of their invasiveness and resistance to surgical procedures as well as chemo- and radiotherapy. ${ }^{[6]}$ The urgent need for novel therapies has led to great emphasis on the development of new anticancer drugs including nanoparticles as cytostatic drug delivery vehicles.

Nanoparticles are structures between one and several hundred nanometers in diameter. There are three major physical properties of nanoparticles: (i) they are highly mobile in the free state; (ii) they have large surface areas; and (iii) they may exhibit quantum effects due to the movement of electrons. They have unique material characteristics, and manufactured nanoparticles may find practical applications in a variety of areas, including medicine. The nanoparticlemediated targeted delivery of drugs might significantly reduce the dosage required, increase drug specificity and bioavailability, overcome chemoresistance and reduce side effects.

The history of therapeutic nanoparticles began in the 1950s with a polymer-drug conjugate designed by Jatzkewitz, followed by Bangham who discovered the liposomes in mid-1960s. In 1972, Scheffel and colleagues first reported albumin based nanoparticles, which formed the basis of albumin-bound paclitaxel (Abraxane) ${ }^{[7]}$

Targeted delivery in cancer therapy is an important challenge for oncologists. Nanovectors for drug delivery typically contain a core material or matrix, a therapeutic payload, and surface modifications in some cases. Possible advantages of nanoparticle delivery systems over conventional anticancer chemotherapy include: (i) protection of drugs from degradation in the body; (ii) enhanced absorption into tumor cells; and (iii) decreased interaction of drugs with normal cells. ${ }^{[8]}$ Ideal properties of nanoparticles for drug delivery are shown in Table 1. Nano-based drug delivery carriers, or nanocarriers, can consist of a wide variety of materials, both organic (polymeric, lipid, protein, or viral) and inorganic. The largest nanocarriers are liposomes (80-200 nm diameter), polymeric nanoparticles (40$100 \mathrm{~nm}$ ) or micelles $(20-60 \mathrm{~nm})$; the smallest ones are dendrimers $\left(<10 \mathrm{~nm}\right.$ diameter) ${ }^{[9]}$ There have been several reports describing the delivery of multiple anticancer agents using nanocarriers, some having been evaluated in clinical trials. Some nanodrugs have been FDA approved. ${ }^{[10]}$ The approved nanodrugs for anticancer therapy are given in Table 2 .

The blood-brain barrier (BBB) protects brain neural tissues and works as a diffusion barrier that impedes the influx of toxins and other compounds, including

Table 1: Ideal properties of nanoparticles for drug delivery. Modified from ${ }^{[78,79]}$

\begin{tabular}{l}
\hline Ideal properties of nanoparticles for drug delivery \\
\hline Non-toxic \\
Biocompatible \\
Biodegradable \\
Physically stable in blood \\
Prolonged time in circulation \\
Non-immunogenic/non-activating neutrophils/non-inflammatory \\
Non-trombogenic/non-agregating platelets \\
Avoidance of reticuloendothelial system \\
Amenable to small molecules, peptides, proteins and nucleic acids \\
Inexpensive/easy manufacturing \\
\hline
\end{tabular}


Table 2: FDA-approved anticancer nanodrugs. Modified from ${ }^{[80]}$

\begin{tabular}{llll}
\hline Name & Description & Indication & Approval (year) \\
\hline DaunoXome & Liposomal daunorubicin & HIV-releated Kaposi sa & FDA 96 \\
DepoCyt & Liposomal cytarabin & Lymphomatous meningitis & FDA 96 \\
Oncaspar & PEG asparaginase & Acute lymphoblastic leukemia & FDA 94 \\
Abraxane & Albumin-bound paclitaxel nanospheres & Various cancers & FDA 05 EMEA 08, FDA 13 \\
& & Pancreatic ca & Europe + Canada \\
Myocet & Liposomal doxorubicin & Breast ca & FDA 12 \\
Marqibo & Liposomal vincristin & Acute lymphoblastic leukemia & Europe + Korea \\
Genexol & Paclitaxel loaded polymeric micelle & Breast ca, small cell lung ca & FDA 15 \\
Onivyde & Liposomal irinotecan & Pancreatic ca & \\
\hline
\end{tabular}

sa: sarcoma; ca: carcinoma

drugs, from blood to the brain. ${ }^{[11]}$ Its main components are brain endothelial cells, basal membranes, pericytes embedded in the basal membrane, and astrocytic endfeet. The BBB is characterized by the presence of tight intercellular junctions, minimal pinocytotic activity, and a lack of fenestrations, qualities that distinguish BBB endothelial cells from peripheral cells. Endogenous and exogenous compounds including drugs may cross the BBB by passive diffusion, carrier-mediated transport, endocytosis, or active transport. The efflux and influx transporters of BBB comprise transporters like ATP-binding cassette transporters and solute carrier transporters. ${ }^{[12]}$ The different types of transport across the BBB are shown in Figure 1.

The inability of drugs to cross the BBB is one of the major impairments to developing treatments for neurological diseases. ${ }^{[13-16]}$ This highly restrictive, physiologic barrier prevents $98 \%$ of small-molecule drugs and virtually $100 \%$ of large-molecule drugs from reaching the central nervous system from blood circulation. Numerous methods to bypass the BBB have been investigated, such as transient disruption of the BBB, inhibition of efflux pumps, or transport using endogenous transcytosis systems, including receptormediated transcytosis. Nanodrugs are another approach to overcoming this obstacle to brain tumor treatment.

This review presents a comprehensive overview of preclinical in vitro and in vivo research and clinical studies of nanodrugs in therapy of brain tumors.

\section{NANOCARIERS FOR ANTICANCER DRUGS}

Drug nanodelivery has gained a great deal of attention from researchers. ${ }^{[17-19]}$ However, some difficulties related to drug delivery may occur, such as troublesome solubility and biological availability, short time in circulation, and inconvenient biodistribution to the target organ. The key features of anticancer nanoparticles are principally large size, surface properties (e.g. hydrophobicity), and in some cases also targeting ligands. The development of a broad range of nanoparticles with varying size, composition, and functionality has provided a significant resource for nanomedicine.

Although nanoparticles avoid renal clearance, they tend to accumulate in the mononuclear phagocyte system (MPS). ${ }^{[20]}$ Surface conjugation with polyethylene glycol (PEG) and other polymers improves particle

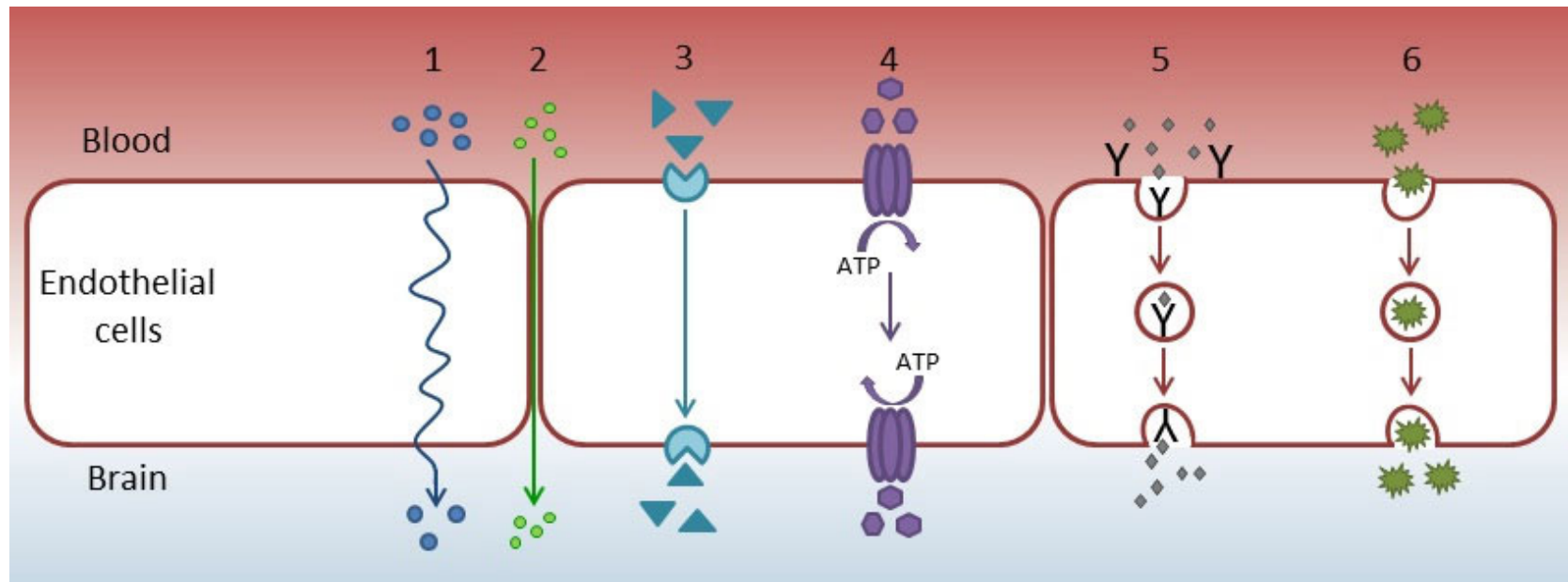

Figure 1: Mechanisms of transport across the blood-brain barrier. (1) Transcellular diffusion (small hydrophobic molecules); (2) paracellular diffusion (small water soluble molecules); (3) carrier-mediated transport (e.g. glucose, amino acids, vinca alkaloids); (4) active efflux transport; (5) receptor-mediated transport (e.g. insulin, leptin, transferrin); (6) adsorptive-mediated endocytosis (e.g. albumin, plasma proteins). ATP: adenosine triphosphate 
circulation by reducing uptake into the MPS. The requirements for nanoparticle properties also depend on tumor characteristics, including cancer type, stage of disease, and location. Delivering multiple agents in vivo is complicated because of their independent pharmacokinetics, biodistribution, and clearance. A delivery system also has to transport a drug with high efficiency to target cells, with minimal toxicity and immune response. Drug toxicity can be reduced by encapsulating the free drug (e.g. liposomes) or by local activation of a pro-drug. ${ }^{[21]}$

Nanoparticles designed for cancer therapy consist of various components, generally a nanocarrier and an active agent. ${ }^{[22]}$ Drug-carrier nanoparticles are considered as submicroscopic colloidal systems that may act as drug vehicles, either as nanospheres (the matrix system in which the drug is dispersed) or nanocapsules (reservoirs in which the drug is confined in hydrophobic or hydrophilic core surrounded by a single polymeric membrane). ${ }^{[23]}$

Nanoparticles as carriers for anticancer drugs make them promising candidates to overcome chemoresistance of cancer cells, because nanoparticles loaded by cytostatic drugs promote their cellular uptake and considerably decrease their efflux, prolong drug systemic circulation lifetime, and enable targeted drug delivery. ${ }^{[2]}$ These particles can be modified with various types of materials including biomolecules. Altering the organizations of atoms can modify the properties of nanoparticles, such as elasticity, plasticity, strength, and conductivity.

Nanoparticle systems have unique properties that allow for both passive and active targeting of tumors. ${ }^{[27]}$ Tumor neovasculature has abnormal architecture and vessels are highly permeable. The tumor mass has also poor lymphatic drainage, allowing for accumulation of macromolecules greater than approximately $40 \mathrm{kDa}$ within its microenvironment. Nanoparticles utilize this feature, known as the enhanced permeability and retention (EPR) effect, to target solid tumors. The ideal size range to benefit from the EPR effect is between 10 and $200 \mathrm{~nm}$. Outside this range, smaller particles will be cleared by the kidney, preventing accumulation within the tumor site, while larger particles will not adequately penetrate the tumor vasculature and interstitial space. However, some clinical trials have not shown the efficacy of the EPR effect. ${ }^{[28]}$ One possible cause of EPR effect failure could be increased interstitial pressure in the tumor microenvironment. It has also been assumed that the EPR effect cannot be employed after an operation. Attempts have been made to increase the efficiency of the EPR effect by induction of hypertension, by repairing the abnormal vasculature, or by targeting of perivascular cells. ${ }^{[28]}$

\section{Targeting molecules}

Active targeting, i.e. surface modification of nanoparticles, is a method to decrease uptake in normal tissue and increase accumulation in a tumor. Strategies for active targeting of tumors usually involve targeting surface membrane proteins that are upregulated in cancer cells. ${ }^{[25]}$ Targeting molecules are typically antibodies or their fragments, aptamers, small molecules, or oligopeptides. Nanoparticles coupled with surface ligands or antibodies can localize to tissue, expressing the associated receptors or antigens and improving delivery efficacy. ${ }^{[10]}$ Some ligand receptor interactions will facilitate receptor-mediated endocytosis, further enhancing payload delivery. Surface ligand or antibody coupling can achieve densities high enough to interact efficiently with target sites, qualities well suited to cancer therapies.

Monoclonal antibodies, particularly lgG, are frequently used for targeting. Antigen binding sites represent only a small part of the overall size of antibodies. $F(a b) 2$ fragments retain both antigen binding sites of the antibody, coupled by disulfide linkages. Many tumors up-regulate growth factor receptors, such as HER2/ neu in certain breast cancers, which can be targeted with anti-HER2/neu surface antibodies. ${ }^{[29]}$ Liposomes modified with monoclonal antibodies against glial fibrillary acidic proteins or human insulin receptors have been studied to determine if they cross the BBB. ${ }^{[30]}$ Transferrin receptor (TfR) is another primary target investigated for receptor-mediated transcytosis across the BBB because of its high expression on BBB endothelium. ${ }^{[31]}$

Aptamers are folded single strand oligonucleotides, 25-100 nucleotides in length, that bind to molecular targets. ${ }^{[32]}$ For example, EpCAM-fluoropyrimidine RNA aptamer-modified doxorubicin-loaded PLGAb-PEG nanoparticles, which bond specifically to the extracellular domain of epithelial-cell adhesion molecules, have been investigated in non-small lung cancer model. Aptamer-conjugated nanoparticles in vitro have displayed increased cytotoxicity and decreased volume of xenografts compared with nontargeted nanoparticles.

Small molecules used for targeting include peptides, growth factors, carbohydrates and receptor ligands. Specific examples of small molecules include folic acid, transferrin and the RGD peptides. Example of small-molecule targeting protein is an HER2/ neu ligands (AHNP) for targeting of poly (lactidecoglycolide) nanoparticles with docetaxel, which has 
been investigated in vitro with HER2+ breast cancer cells. ${ }^{[33]}$

Folic acid (FA) is essential for DNA synthesis, DNA repair, and methylation of DNA and is therefore necessary for cell survival and proliferation. The human folate receptor (FR), a glycosylphosphatidylinositolanchored membrane protein of $38 \mathrm{kDa}$, has high affinity for $F A$, and is currently considered an essential component in the cellular accumulation of FA required in chemotherapy. FR expression is very low or undetectable in most normal cells and tissues, but it is upregulated in ovarian, breast, brain, lung, colorectal cancers as well as brain tumors. ${ }^{[34,35]}$ Through the process of endocytosis, ligand-bound receptor is internalized and released from the receptor through

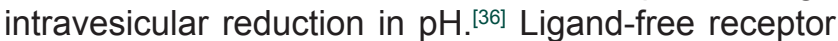
is then recycled to the cell surface. Interestingly, covalent conjugation of small molecules, proteins and even liposomes to the gamma-carboxyl moiety of FA does not alter FA ability to bind to the FR and undergo endocytosis by receptor bearing cells. FR-mediated liposomal delivery has been shown to enhance the antitumor efficacy of doxorubicin both in vitro and in vivo, and to overcome P-glycoprotein-mediated multidrug resistance. ${ }^{[37]}$

Transferrin (Tf) is a single-chain iron-transporting glycoprotein that supplies iron into cells via receptormediated endocytosis. The TfR is expressed at low levels in most normal tissues but is overexpressed in many tumor types. The crucial aspect of Tf for molecular targeting applications, the binding of Tf to TfR on the external surface of tumor cells, is 10 times to 100 times more effective in tumor cells than in normal cells. ${ }^{[38]}$ Drug delivery systems can take advantage of this feature, most often by labeling the surface of the drug carrier with Tf, which is recognized by, and actively transported into, tumor cells. Therefore, Tfmodified liposomes, nanoparticles and dendrimers have been widely investigated in recent years. Despite the perceived potential of anti-TfR antibody-drug conjugates, a BBB-permeable drug using this approach has not yet been introduced for clinical use. ${ }^{[16]}$

Ferritin protein also self-assembles naturally into a hollow nanocage called apoferritin, useful for encapsulation of any molecule of interest. ${ }^{[39]}$ Apoferritin can be modified with recognition ligands to achieve tumor-specific targeting. These extra surface modifications can avoid renal clearance and ensure EPR effect; however, they also eliminate the intrinsic tumor-specific binding of natural ferritin and disturb its in vivo performance and biocompatibility due to altered surface physicochemical properties of ferritin.
The authors have studied antibody targeted apoferritin mediated transport of doxorubicin, in which the surface of apoferritin can be modified with antibodies to enhance its targeting ability. These studies compared the cytotoxic effect of doxorubicin-loaded apoferritin, with and without surface targeting antibody anti-GCPII (PSMA), with that of free doxorubicin in vitro on prostatic cancer cell line ( $\mathrm{LNCaP}$ ) expressing PSMA as well as human umbilical vein endothelial cells (HUVEC) as a model of nonmalignant cells. The effect of doxorubicinloaded apoferritin nanocarriers on cancer and healthy cells was similar to that of free doxorubicin. However, the real-time impedance-based platform demonstrated lower toxicity to HUVEC with doxorubicin loaded apoferritin than with free doxorubicin [Figure 2]. Entry of doxorubicin-loaded apoferritin nanocarriers with and without targeting antibody was higher into LNCaP than into HUVEC (Cerna et al., unpublished results).

Oligopeptides are also molecules used for targeting. The RGD (Arg-Gly-Asp) oligopeptide is a component of the extracellular matrix protein fibronectin and promotes cell adhesion and regulates migration, growth, and proliferation. ${ }^{[25,40]}$ RGD is known to serve as a recognition motif in multiple ligands for several different integrins. RGD-containing peptide can be internalized into cells by integrin-mediated endocytosis. Recently, integrin-mediated carriers have been investigated as gene vehicles to enhance gene transfection and as vehicles to deliver anticancer agents. The upregulation of integrins is known to be promoted by angiogenic factors in several cancer types.

\section{NANOPARTICLES IN THERAPY OF BRAIN TUMORS}

Nanoparticles represent one of the possibilities of overcoming the BBB and delivering anticancer drugs to the brain. Therapy for brain tumors, particularly glioblastoma, using nanoparticles has been the subject of several preclinical experiments and clinical studies, but no nanodrug is as yet approved for brain tumor therapy.

\section{Preclinical studies in brain tumors}

Lipid nanoparticles loaded with doxorubicin have been investigated as a potential drug carrier to the brain, although doxorubicin cannot cross the BBB. The pharmacokinetics and tissue distribution of doxorubicin were studied in healthy rats, using i.v. administration of either free doxorubicin or doxorubicin incorporated into solid lipid nanoparticles (NANO DOX) in equivalent doses. ${ }^{[42]}$ Several blood samples and tissue samples of liver, spleen, heart, lung, kidney, and brain were collected. The mean peak plasma concentrations of 


\section{LNCaP}

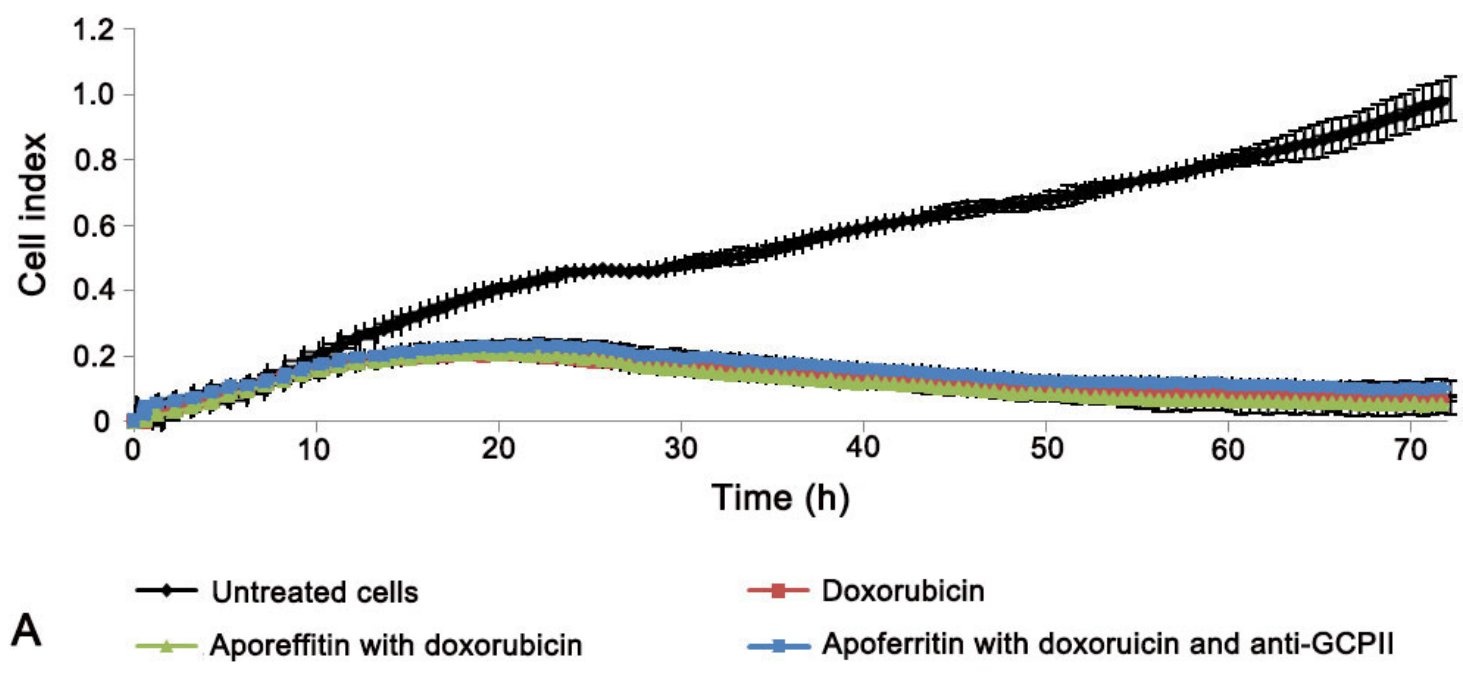

HUVEC

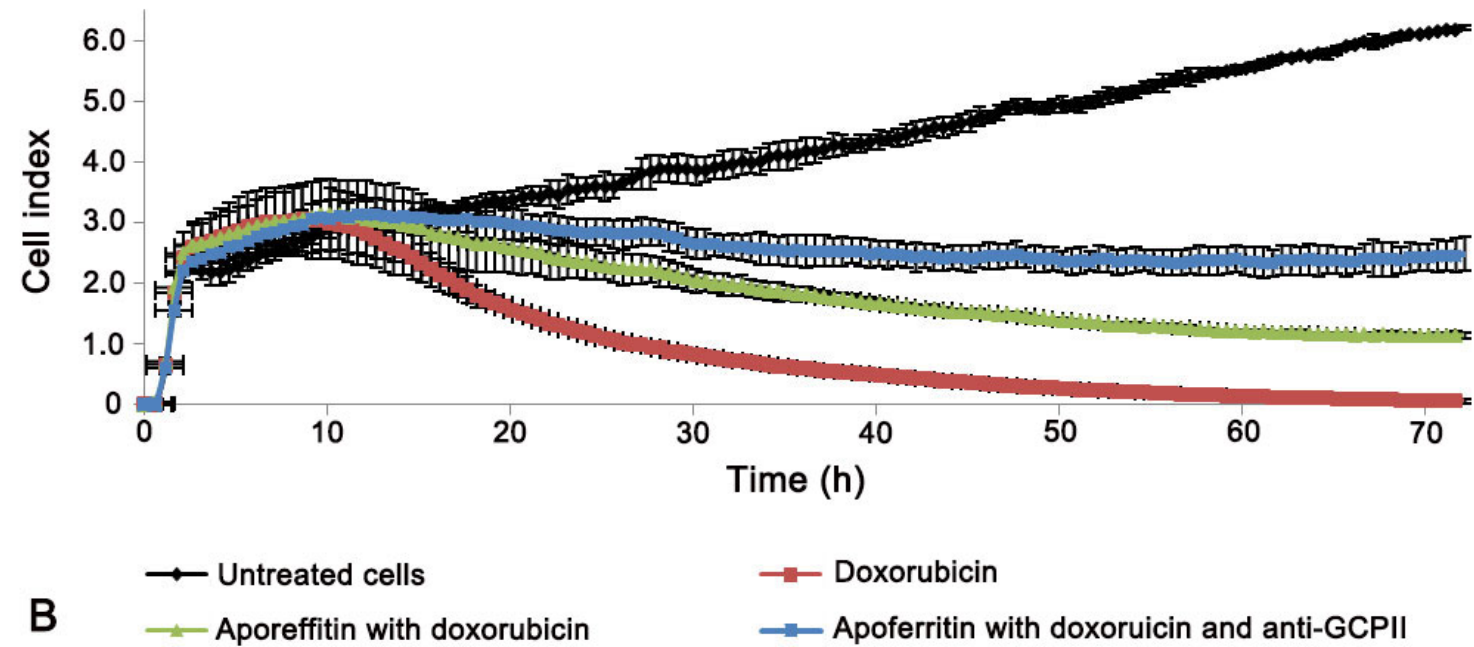

Figure 2: Cytotoxic effect of doxorubicin loaded apoferritin with and without targeting antibody anti-GCPII (PSMA) on its surface and free doxorubicin on (A) prostatic cancer cell line (LNCaP) expressing PSMA and (B) human umbilical vein endothelial cells (HUVEC)

free doxorubicin were lower than after NANO DOX treatment. In all rat tissues except the brain, the amount of doxorubicin was always lower after the injection of NANO DOX than after the injection of free doxorubicin. In the brain, however, NANO DOX increased the doxorubicin concentration significantly. The same study design, repeated in healthy rabbits, showed similar pharmacokinetic behavior and tissue distribution parameters. ${ }^{[43]}$ Docetaxel-incorporated albumin-lipid nanoparticles (DNPs) in vitro induce apoptosis of several cancer cell lines, and in vivo, accumulate at the experimental glioma site. ${ }^{[4]}$ This phenomenon is believed to be due to EPR effect. Liposomes containing temozolomide (TMZ) combined with anti-transferrin receptor single-chain antibody fragments were found to be more effective than free TMZ in both TMZ-resistant and TMZ-sensitive glioblastoma cells in mouse models. ${ }^{[45]}$ Moreover, these liposomes showed significantly reduced toxicity. These results show that these liposomes may be an efficient vehicle for delivering BBB-impermeable drugs to the brain.

Biodegradable polymer-based nanoparticles and gold nanoparticles have both shown promise for delivering drugs across the BBB to treat glioma. ${ }^{[46]}$ Gromnicova et al. ${ }^{[4]}$ found that glucose-coated gold nanoparticles cross brain endothelium three times faster than non-brain endothelium. Huwyler et al. ${ }^{[4]}$ investigated daunorubicinloaded liposomes with anti-transferrin receptor antibody, using an animal model, and found increased brain daunorubicin concentration compared with free drug. 
Nanoparticles show promise for specific and efficient intracerebral delivery of drugs for the treatment of glioma. ${ }^{[49]}$ A two-dose regimen of topotecan nonPEGylated liposomes, locally administered with paramagnetic gadodiamide nanoparticles, increased survival rates in a U87MG glioblastoma intracranial xenograft model compared with controls; the effect was topotecan dose-dependent. ${ }^{[50]}$

Gadolinium nanoparticles enhance MRI monitoring and are well tolerated. These nanoparticles can penetrate the BBB and be uptaken by the brain tumor parenchyma. ${ }^{[51]}$ Metal nanoparticles are also frequently integrated with other techniques such as microwaveinduced hyperthermia to further increase their cellular transduction. ${ }^{[52]}$ The $\alpha$-helical right handed coiled coils associated with platinum (PtIV) compound showed higher toxicity to human malignant glioma cells compared with free Pt(IV) in vitro and in vivo, without affecting healthy astrocytes in vitro. ${ }^{[53]}$

Carrier-mediated transport (CMT) can transport small molecules from the blood to the brain. Receptor-mediated transport (RMT) systems are expressed on the BBB and provide transport of large endogenous biomolecules ${ }^{[54]}$ [Figure 1]. During RMT, macromolecules move across the endothelial cells into the brain, due to the expression of several peptidespecific receptors, e.g. neonatal Fc receptor, ${ }^{[55]}$ lowdensity lipoprotein receptor-related protein receptor, transferrin receptor, ${ }^{[56]}$ lactoferrin receptor, ${ }^{[57]}$ and insulin receptor. ${ }^{[58]}$ Some of the above-mentioned receptors have been used for drug delivery as a molecular "Trojan horse". Shilo et al. ${ }^{[59]}$ demonstrated that insulin-targeted gold nanoparticles cross the BBB after systemic administration.

Gao et al. ${ }^{[60]}$ investigated transferrin-folate doxorubicinloaded liposomes. The amount of doxorubicin transported across the BBB in the transferrin-folate doxorubicin-loaded liposome group of glioma bearing rates was sevenfold higher than in the non-targeted doxorubicin-loaded liposome-treated group. Boado et al. ${ }^{[61]}$ found that fused lysosomal enzyme with anti-human insulin receptor monoclonal antibody could deliver fusion protein across the BBB at therapeutic levels, while free lysosomal enzyme did not cross the BBB. Yang et al. ${ }^{[62]}$ tested dual peptide-modified (using lowdensity lipoprotein receptor-related protein receptor and neuropilin-1 receptor) liposomes loaded with vascular endothelial growth factor siRNA and docetaxel; the target was human glioblastoma xenografts in mice. These dual-modified liposomes showed the highest uptake compared with single modified or non-modified liposomes.
In anotherstudy, cetylalcohol/polysorbate nanoparticles loaded with paclitaxel were more cytotoxic to glioblastoma cells and had higher brain uptake in an experimental animal model than paclitaxel alone. ${ }^{[63]}$ The investigators speculated that nanoparticles may limit binding of paclitaxel to p-glycoprotein, causing higher brain and tumor cell uptake.

Coated poly (butylcyanoacrylate) (PBCA) nanoparticles have been studied as a delivery system for drugs in the brain. ${ }^{[64,65]}$ Polysorbate 80 was found to be the most efficient modifier of nanoparticles. Transport across the BBB of polysorbate 80-coated nanoparticles has been presumed to involve receptor-mediated endocytosis by endothelias. Polysorbate 80 absorbs plasmatic apolipoprotein $E$ (Apo-E) and nanoparticles coated with Apo-E are internalized by the LDL uptake system. ${ }^{[66]}$ In one study in rats, PBCA nanoparticles with doxorubicin increased brain doxorubicin concentrations to levels more than 60 times that of free drug, while heart levels were very low. ${ }^{[67]}$ In another rat brain model, polysorbate 80 coated poly-lactic-co-glycolic acid nanoparticles loaded with methotrexate-transferrin conjugates were investigated and showed better penetration, lower organ toxicity and higher anti-tumor activity as compared with non-targeting nanoparticles. ${ }^{[68]}$

Doxorubicin bound to polysorbate-coated nanoparticles was associated with significantly longer survival of glioblastoma-bearing rats compared with groups treated with free doxorubicin or noncoated nanoparticles with doxorubicin. ${ }^{[69]}$ Poly-lactic-co-glycolic acid (PLGA) camptothecin-loaded nanoparticles were investigated in orthotopic murine glioma. Nanoparticles were well tolerated and effective against glioma. ${ }^{[0]}$ Cetuximabmagnetic iron-oxide nanoparticles (IONP) that bind to both wild-type EGFR+ and mutated EGFR+ patient-derived glioblastoma cells are internalized by tumor cells and promote internalization of the EGFR, resulting in enhanced apoptosis. Treatment with cetuximab-IONPs proved efficacious in orthotopic glioblastoma xenografts in mouse and rats, and showed a favorable safety profile, as no toxicity to healthy immunocompetent mice was observed. ${ }^{[71]}$

The in vitro and in vivo studies described above seem promising for the treatment of brain tumors, particularly glioblastoma, the tumor with the worst prognosis. The inclusion of the most efficacious and safe nanoparticles designed for cancer therapy in clinical studies is warranted. Nevertheless, despite the successful results of preclinical experiments, the progress in applying these strategies in brain tumors is still modest when compared with treatments in other types of tumors. 


\section{Clinical studies in brain tumors}

A phase I clinical study of paclitaxel-Angiopep-2 peptide-drug conjugate that binds to the low-density lipoprotein receptor-related protein-1 receptor (GRN1005) has been carried out in patients with recurrent glioma grade 2-4. The clinical data show that GRN1005 facilitated the penetration of paclitaxel into tumor tissue. ${ }^{[72]}$ However, interim analysis of the phase II trial did not show therapeutic response. ${ }^{[73]}$

Transferrin conjugated with diphteric toxin (Tf-CRM107) demonstrated in vitro and in vivo toxicity to glioma cells and was effective when administrated locally to xenografts. Using local administration, low toxicity and tumor response were demonstrated in patients with recurrent high grade brain tumors in phase I and II clinical trials. The response rate was $35 \%$ and overall survival of responders was 74 weeks. ${ }^{[7]}$ Unfortunately, an early phase III clinical trial using this therapy had to be terminated due to disappointing preliminary results. ${ }^{[75]}$

In a clinical study of liposomal doxorubicin in patients with high-grade gliomas, Fabel et al. ${ }^{[75]}$ found improved overall survival than in past trials using conventional therapies. Hau et al. ${ }^{[77]}$ demonstrated that pegylated liposomal doxorubicin in patients with recurrent highgrade glioma was efficacious and well tolerated.

These results presented above suggest that some nanodrugs may be efficient in therapy of high grade brain tumors, a topic of great potential interest for clinicians.

\section{FUTURE DIRECTIONS AND CONCLUSIONS}

Although the available clinical trial data are limited, evidence suggests that nanoparticles have potential_in diagnosis, operative management and adjuvant therapy for brain tumors. Because the field of nanotechnology is young, the long-term health effects of nanoparticles are currently unknown. More study of nanoparticle biodistribution, pharmacokinetics, toxicity and role in therapeutic protocols is warranted if nanoparticles are to attain regular clinical use.

\section{Financial support and sponsorship}

Preclinical research of nanodrugs is supported by GACR (NANOCHEMO 14-8344S) and by the Ministry of Health of the Czech Republic for conceptual development of research organization 00064203 (University Hospital Motol, Prague, Czech Republic).

\section{Conflict of interest}

There are no conflicts of interest.

\section{Patient consent}

No patient involved.

\section{Ethics approval}

This article does not contain any studies with human participants or animals.

\section{REFERENCES}

1. Louis DN, Ohgaki H, Wiestler OD, Cavenee WK, Burger PC, Jouvet A, Scheithauer BW, Kleihues P. The 2007 WHO classification of tumours of the central nervous system. Acta Neuropathol 2007;114:97-109.

2. Dolecek TA, Propp JM, Stroup NE, Kruchko C. CBTRUS statistical report: primary brain and central nervous system tumors diagnosed in the United States in 2005-2009. Neuro Oncol 2012;14:1-49.

3. Wilson TA, Karajannis MA, Harter DH. Glioblastoma multiforme: State of the art and future therapeutics. Surg Neurol Int 2014;5:64.

4. Rivkin M, Kanoff RB. Metastatic brain tumors: current therapeutic options and historical perspective. $J$ Am Osteopath Assoc 2013;113:418-23.

5. Fokas E, Steinbach JP, Rödel C. Biology of brain metastases and novel targeted therapies: time to translate the research. Biochim Biophys Acta 2013;1835:61-75.

6. Liu C, Zong H. Developmental origins of brain tumors. Curr Opin Neurobiol 2012;22:844-9.

7. Aslan B, Ozpolat B, Sood AK, Lopez-Berestein G. Nanotechnology in cancer therapy. J Drug Target 2013;21:904-13.

8. Bhowmik A, Khan R, Ghosh MK. Blood brain barrier: a challenge for effectual therapy of brain tumors. Biomed Res Int 2015;320941.

9. Dostalova S, Heger Z, Kudr J, Vaculovicova M, Adam V, Stiborova M, Eckschlager T, Kizek R. Apoferritin: protein nanocarrier for targeted delivery. In: Naik J, editor. Nano Based Drug Delivery. Zagerb: IAPC Publishing; 2015. p. 217-33.

10. Ediriwickrema A, Saltzman WM. Nanotherapy for cancer: targeting and multifunctionality in the future of cancer therapies. ACS Biomater Sci Eng 2015;1:64-78.

11. Hawkins BT, Davis TP. The blood-brain barrier/neurovascular unit in health and disease. Pharmacol Rev 2005;57:173-85.

12. Bhowmik A, Khan R, Ghosh MK. Blood brain barrier: a challenge for effectual therapy of brain tumors. Biomed Res Int 2015;2015:320941.

13. Rapoport SI. Modulation of blood-brain barrier permeability. J Drug Target 1996;3:417-25.

14. Tsuji A, Tamai I. Sodium- and chloride-dependent transport of taurine at the blood-brain barrier. Adv Exp Med Biol 1996;403:385-91.

15. Groothuis DR. The blood-brain and blood-tumor barriers: a review of strategies for increasing drug delivery. Neuro Oncol 2000;245-59.

16. Clark AJ, Davis ME. Increased brain uptake of targeted nanoparticles by adding an acid-cleavable linkage between transferrin and the nanoparticle core. Proc Natl Acad Sci U S A 2015;112:12486-91.

17. Patra CR, Bhattacharya R, Mukhopadhyay D, Mukherjee P. Fabrication of gold nanoparticles for targeted therapy in pancreatic cancer. Adv Drug Deliv Rev 2010;62:346-61.

18. Gu FX, Karnik R, Wang AZ, Alexis F, Levy-Nissenbaum E, Hong S, Langer RS, Farokhzad OC. Targeted nanoparticles for cancer therapy. Nano Today 2007;2:14-21.

19. Chomoucka J, Drbohlavova J, Huska D, Adam V, Kizek R, Hubalek J Magnetic nanoparticles and targeted drug delivering. Pharmacol Res 2010;62:144-9.

20. Adiseshaiah PP, Hall JB, McNeil SE. Nanomaterial standards for efficacy and toxicity assessment. Wiley Interdiscip Rev Nanomed Nanobiotechnol 2010;2:99-112.

21. Kanthamneni N, Sharma S, Meenach SA, Billet B, Zhao JC, Bachelder 
EM, Ainslie KM. Enhanced stability of horseradish peroxidase encapsulated in acetalated dextran microparticles stored outside cold chain conditions. Int J Pharm 2012;431:101-10.

22. Ferrari M. Cancer nanotechnology: opportunities and challenges. Nat Rev Cancer 2005;5:161-71.

23. Juillerat-Jeanneret L. The targeted delivery of cancer drugs across the blood-brain barrier: chemical modifications of drugs or drugnanoparticles? Drug Discov Today 2008;13:1099-106.

24. Drbohlavova J, Chomoucka J, Adam V, Ryvolova M, Eckschlager T, Hubalek J, Kizek R. Nanocarriers for anticancer drugs -- new trends in nanomedicine. Curr Drug Metab 2013;14:547-64.

25. Dawidczyk CM, Russell LM, Searson PC. Nanomedicines for cancer therapy: state-of-the-art and limitations to pre-clinical studies that hinder future developments. Front Chem 2014;2:69.

26. $\mathrm{Hu} \mathrm{C}-\mathrm{MJ}$, Zhang L. Therapeutic nanoparticles to combat cancer drug resistance. Curr Drug Metab 2009; 10:836-41.

27. Danhier F, Feron O, Préat V. To exploit the tumor microenvironment: Passive and active tumor targeting of nanocarriers for anti-cancer drug delivery. J Control Release 2010;148:135-46.

28. Huynh E, Zheng G. Cancer nanomedicine: addressing the dark side of the enhanced permeability and retention effect. Nanomedicine (Lond.) 2015;10:1993-5

29. Peer D, Karp JM, Hong S, Farokhzad OC, Margalit R, Langer R. Nanocarriers as an emerging platform for cancer therapy. Nat Nanotechnol 2007;2:751-60.

30. Pardridge WM. Vector-mediated drug delivery to the brain. Adv Drug Deliv Rev 1999;36:299-321.

31. Bray N. Biologics: Transferrin' bispecific antibodies across the bloodbrain barrier. Nat Rev Drug Discov 2015;14:14-5.

32. Alibolandi M, Ramezani M, Abnous K, Sadeghi F, Atyabi F, Asouri $\mathrm{M}$, Ahmadi AA, Hadizadeh F. In vitro and in vivo evaluation of therapy targeting epithelial-cell adhesion-molecule aptamers for nonsmall cell lung cancer. J Control Release 2015;209:88-100.

33. Yang Z, Tang W, Luo X, Zhang X, Zhang C, Li H, Gao D, Luo H, Jiang Q, Liu J. Dual-ligand modified polymer-lipid hybrid nanoparticles for docetaxel targeting delivery to Her2/neu overexpressed human breast cancer cells. J Biomed Nanotechnol 2015;11:1401-17.

34. Shan L, Liu M, Wu C, Zhao L, Li S, Xu L, Cao W, Gao G, Gu Y. Multi-small molecule conjugations as new targeted delivery carriers for tumor therapy. Int J Nanomedicine 2015;10:5571-91.

35. Assaraf YG, Leamon CP, Reddy JA. The folate receptor as a rational therapeutic target for personalized cancer treatment. Drug Resist Updat 2014;17:89-95.

36. Goren D, Horowitz AT, Tzemach D, Tarshish M, Zalipsky S, Gabizon A. Nuclear delivery of doxorubicin via folate-targeted liposomes with bypass of multidrug-resistance efflux pump. Clin Cancer Res 2007;6:1949-57.

37. Pan X, Lee RJ. Tumor-selective drug delivery via folate receptortargeted liposomes. Expert Opin Drug Deliv 2004;1:7-17.

38. Guo L, Zhang H, Wang F, Liu P, Wang Y, Xia G, Liu R, Li X, Yin $\mathrm{H}$, Jiang $\mathrm{H}$, Chen B. Targeted multidrug-resistance reversal in tumor based on PEG-PLL-PLGA polymer nano drug delivery system. Int J Nanomedicine 2015;10:4535-47.

39. Liang M, Fan K, Zhou M, Duan D, Zheng J, Yang D, Feng J, Yan X. H-ferritin-nanocaged doxorubicin nanoparticles specifically target and kill tumors with a single-dose injection. Proc Natl Acad Sci 2014; 111:14900-5

40. Cao Y, Zhou Y, Zhuang Q, Cui L, Xu X, Xu R, He X. Anti-tumor effect of RGD modified PTX loaded liposome on prostatic cancer. Int $J$ Clin Exp Med 2015;8:12182-91.

41. Orringer DA, Koo YE, Chen T, Kopelman R, Sagher O, Philbert MA. Small solutions for big problems: the application of nanoparticles to brain tumor diagnosis and therapy. Clin Pharmacol Ther 2009;85:531-4.
42. Fundarò A, Cavalli R, Bargoni A, Vighetto D, Zara GP, Gasco MR. Non-stealth and stealth solid lipid nanoparticles (SLN) carrying doxorubicin: pharmacokinetics and tissue distribution after i.v. administration to rats. Pharmacol Res 2000;42:337-43.

43. Zara GP, Cavalli R, Bargoni A, Fundarò A, Vighetto D, Gasco MR. Intravenous administration to rabbits of non-stealth and stealth doxorubicin-loaded solid lipid nanoparticles at increasing concentrations of stealth agent: pharmacokinetics and distribution of doxorubicin in brain and other tissues. J Drug Target 2002;10:327-35.

44. Gao H, Cao S, Yang Z, Zhang S, Zhang Q, Jiang X. Preparation,characterization and anti-glioma effects of docetaxel-incorporated albumin-lipid nanoparticles. J Biomed Nanotechnol 2015;11:2137-47.

45. Kim SS, Rait A, Kim E, DeMarco J, Pirollo KF, Chang EH Encapsulation of temozolomide in a tumor-targeting nanocomplex enhances anti-cancer efficacy and reduces toxicity in a mouse model of glioblastoma. Cancer Lett 2015;369:250-8.

46. Auffinger B, Thaci B, Nigam P, Rincon E, Cheng Y, Lesniak MS. New therapeutic approaches for malignant glioma: in search of the Rosetta stone. F1000 Med Rep 2012;4:18.

47. Gromnicova R, Davies HA, Sreekanthreddy P, Romero IA, Lund T, Roitt IM, Phillips JB, Male DK. Glucose-coated gold nanoparticles transfer across human brain endothelium and enter astrocytes in vitro. PLoS One 2013;8:e81043.

48. Huwyler J, Wu D, Pardridge WM. Brain drug delivery of small molecules using immunoliposomes. Proc Natl Acad Sci U S A 1996;93:14164-9.

49. Krol S. Challenges in drug delivery to the brain: nature is against us. $J$ Control Release 2012;164:145-55.

50. Grahn AY, Bankiewicz KS, Dugich-Djordjevic M, Bringas JR, Hadaczek P, Johnson GA, Eastman S, Luz M. Non-PEGylated liposomes for convection-enhanced delivery of topotecan and gadodiamide in malignant glioma: initial experience. $J$ Neurooncol 2009;95:185-97

51. Kreuter J. Drug delivery to the central nervous system by polymeric nanoparticles: what do we know? Adv Drug Deliv Rev 2014;71:2-14

52. Stockwell J, Abdi N, Lu X, Maheshwari O, Taghibiglou C. Nove central nervous system drug delivery systems. Chem Biol Drug Des 2014;83:507-20

53. Thanasupawat T, Bergen H, Hombach-Klonisch S, Krcek J, Ghavam S, Del Bigio MR, Krawitz S, Stelmack G, Halayko A, McDougall M, Meier M, Stetefeld J, Klonisch T. Platinum (IV) coiled coil nanotubes selectively kill human glioblastoma cells. Nanomedicine 2015;11:913-25.

54. Allhenn D, Boushehri MAS, Lamprecht A. Drug delivery strategies for the treatment of malignant gliomas. Int J Pharm 2012;436:299-310.

55. Sumbria RK, Boado RJ, Pardridge WM. Brain protection from stroke with intravenous TNF $\alpha$ decoy receptor-Trojan horse fusion protein. $J$ Cereb Blood Flow Metab 2012;32:1933-8.

56. Tortorella S, Karagiannis TC. Transferrin receptor-mediated endocytosis: a useful target for cancer therapy. J Membr Biol 2014;247:291-307.

57. Miao D, Jiang M, Liu Z, Gu G, Hu Q, Kang T, Song Q, Yao L, Li W, Gao $\mathrm{X}$, Sun M, Chen J. Co-administration of dual-targeting nanoparticles with penetration enhancement peptide for antiglioblastoma therapy. Mol Pharm 2014;11:90-101.

58. Kuo YC, Shih-Huang CY. Solid lipid nanoparticles carrying chemotherapeutic drug across the blood-brain barrier through insulin receptor-mediated pathway. J Drug Target 2013;21:730-8.

59. Shilo M, Motiei M, Hana P, Popovtzer R. Transport of nanoparticles through the blood-brain barrier for imaging and therapeutic applications. Nanoscale 2014;6:2146-52.

60. Gao JQ, Lv Q, Li LM, Tang XJ, Li FZ, Hu YL, Han M. Glioma targeting and blood-brain barrier penetration by dual-targeting doxorubincin liposomes. Biomaterials 2013;34:5628-39.

61. Boado RJ, Hui EK-W, Lu JZ, Sumbria RK, Pardridge WM. Bloodbrain barrier molecular trojan horse enables imaging of brain uptake of 
radioiodinated recombinant protein in the rhesus monkey. Bioconjug Chem 2013;24:1741-9.

62. Yang ZZ, Li JQ, Wang ZZ, Dong DW, Qi XR. Tumor-targeting dual peptides-modified cationic liposomes for delivery of siRNA and docetaxel to gliomas. Biomaterials 2014;35:5226-39.

63. Koziara JM, Lockman PR, Allen DD, Mumper RJ. Paclitaxel nanoparticles for the potential treatment of brain tumors. $J$ Control Release 2004;99:259-69.

64. Borchard G, Audus KL, Shi F, Kreuter J. Uptake of surfactant-coated poly(methyl methacrylate)-nanoparticles by bovine brain microvessel endothelial cell monolayers. Int J Pharm 1994;110:29-35.

65. Kreuter J, Petrov VE, Kharkevich DA, Alyautdin RN. Influence of the type of surfactant on the analgesic effects induced by the peptide dalargin after its delivery across the blood-brain barrier using surfactant-coated nanoparticles. J Control Release 1997;49:81-7.

66. Kreuter J, Ramge P, Petrov V, Hamm S, Gelprina SE, Engelhardr B, Alyautdin R, Briesen HV, Begley DJ. Direct evidence that polysorbate80-coated poly(butyl cyanoacrylate) nanopaticles deliver drugs to the CNS via specific mechanisms requiring prior binding of the drug to the nanoparticles. Pharm Res 2003;20:409-16.

67. Gulyaev AE, Gelperina SE, Skidan IN, Antropov AS, Kivman GY, Kreuter J. Significant transport of doxorubicin into the brain with polysorbate 80-coated nanoparticles. Pharm Res 1999;16:1564-9.

68. Jain A, Jain A, Garg NK, Tyagi RK, Singh B, Katare OP, Webster TJ, Soni V. Surface engineered polymeric nanocarriers mediate the delivery of transferrin-methotrexate conjugates for an improved understanding of brain cancer. Acta Biomater 2015; 24:140-51.

69. Steiniger SC, Kreuter J, Khalansky AS, Skidan IN, Bobruskin AI, Smirnova ZS, Severin SE, Uhl R, Kock M, Geiger KD, Gelperina SE. Chemotherapy of glioblastoma in rats using doxorubicin-loaded nanoparticles. Int J Cancer 2004;109:759-67.

70. Householder KT, DiPerna DM, Chung EP, Wohlleb GM, Dhruv HD, Berens ME, Sirianni RW. Intravenous delivery of camptothecinloaded PLGA nanoparticles for the treatment of intracranial glioma. Int J Pharm 2015;479:374-80.

71. Kaluzova M, Bouras A, Machaidze R, Hadjipanayis CG. Targeted therapy of glioblastoma stem-like cells and tumor non-stem cells using cetuximab-conjugated iron-oxide nanoparticles. Oncotarget 2015;6:8788-806

72. Drappatz J, Brenner A, Wong ET, Eichler A, Schiff D, Groves MD, Mikkelsen T, Rosenfeld S, Sarantopoulos J, Meyers CA, Fielding RM, Elian K, Wang X, Lawrence B, Shing M, Kelsey S, Castaigne JP, Wen PY. Phase I study of GRN1005 in recurrent malignant glioma. Clin Cancer Res 2013;19:1567-76.

73. Owonikoko TK, Arbiser J, Zelnak A, Shu HK, Shim H, Robin AM, Kalkanis SN, Whitsett TG, Salhia B, Tran NL, Ryken T, Moore MK, Egan KM, Olson JJ. Current approaches to the treatment of metastatic brain tumours. Nat Rev Clin Oncol 2014;11:203-22.

74. Weaver M, Laske DW. Transferrin receptor ligand-targeted toxin conjugate (Tf-CRM107) for therapy of malignant gliomas. $J$ Neurooncol 2003;65:3-13.

75. Arko L, Katsyv I, Park GE, Luan WP, Park JK. Experimenta approaches for the treatment of malignant gliomas. Pharmacol Ther 2010;128:1-36.

76. Fabel K, Dietrich J, Hau P, Wismeth C, Winner B, Przywara S Steinbrecher A, Ullrich W, Bogdahn U. Long-term stabilization in patients with malignant glioma after treatment with liposomal doxorubicin. Cancer 2001;92:1936-42.

77. Hau P, Fabel K, Baumgart U, Rümmele P, Grauer O, Bock A, Dietmaier C, Dietmaier W, Dietrich J, Dudel C, Hübner F, Jauch T, Drechsel E, Kleiter I, Wismeth C, Zellner A, Brawanski A, Steinbrecher A, Marienhagen J, Bogdahn U. Pegylated liposomal doxorubicin-efficacy in patients with recurrent high-grade glioma. Cancer 2004;100:1199-207.

78. Béduneau A, Saulnier P, Benoit JP. Active targeting of brain tumors using nanocarriers. Biomaterials 2007;28:4947-67.

79. Lockman PR, Mumper RJ, Khan MA, Allen DD. Nanoparticle technology for drug delivery across the blood-brain barrier. Drug Dev Ind Pharm 2002;28:1-13.

80. Pillai G. Nanomedicines for cancer therapy: an update of FDA approved and those under various stages of development. SOJ Pharm Pharm Sci 2014;1:13-25. 